\title{
Oophorectomy promotes islet amyloid formation in a transgenic mouse model of Type II diabetes
}

\author{
S. E. Kahn, S. Andrikopoulos, C. B. Verchere, F. Wang, R. L. Hull, J. Vidal
}

Division of Metabolism, Endocrinology and Nutrition, Department of Medicine, University of Washington and VA Puget Sound Health Care System, Seattle, USA

\section{Abstract}

Aims/hypothesis. In Type II (non-insulin-dependent) diabetes mellitus, amyloid depletes islet mass. We previously found that $81 \%$ of male human islet amyloid polypeptide (IAPP) transgenic mice but only $11 \%$ of female mice developed islet amyloid, suggesting that either testosterone promotes or ovarian products protect against amyloid deposition.

Methods. We did a bilateral oophorectomy or sham procedure in female human IAPP transgenic mice ( $n=11$ and $n=8$, respectively) and in female nontransgenic mice ( $n=7$ and $n=9$, respectively) at 6-8 weeks of age. Animals were followed for 1 year on a $9 \%$ fat $(\mathrm{w} / \mathrm{w})$ diet. Before we killed them we measured, fasting plasma human IAPP and did an intraperitoneal glucose tolerance test. Pancreatic content of IAPP and immunoreactive insulin (IRI) were estimated and pancreata were analysed for islet amyloid. Results. No amyloid was detected in either the shamoperated transgenic mice or, as expected, in both groups of non-transgenic mice. In strong contrast, 7 of $11(64 \%)$ oophorectomized mice developed islet amyloid $(p<0.05)$. Amyloid deposition in the oophorectomized transgenic mice was not associated with any differences in incremental body weight, fasting human IAPP concentrations or glucose tolerance between the groups. Furthermore, pancreatic content of mouse IAPP, human IAPP and immunoreactive insulin did not differ between groups.

Conclusion/interpretation. Oophorectomy is associated with an enhancement of islet amyloid formation in the absence of changes in glucose tolerance, circulating IAPP or pancreatic content of IRI, mouse or human IAPP. Thus, the early stages of islet amyloidogenesis seem to be independent of glucose tolerance, with ovarian products having a protective role. [Diabetologia (2000) 43: 1309-1312]

Keywords Islet amyloid, islet amyloid polypeptide, amylin, oophorectomy, oestrogen, glucose tolerance, diabetes, Alzheimer's disease.
Amyloid is present in over $85 \%$ of human subjects with Type II (non-insulin-dependent) diabetes mellitus [1] with islet amyloid polypeptide (IAPP or amylin) being its unique component [2,3]. The amyloidogenic sequence in the mid-portion of the IAPP mole-

Received: 22 March 2000 and in revised form: 25 May 2000

Corresponding author: S. E. Kahn MB, ChB, VA Puget Sound Health Care System (151), 1660 S. Columbian Way, Seattle WA 98108, USA

Abbreviations: FSH, Follicle stimulating hormone; IAPP, Islet amyloid polypeptide/amylin; IAPP-LI, Islet amyloid polypeptide-like immunoreactivity; IPGTT, Intraperitoneal glucose tolerance test; IRI, Immunoreactive insulin. cule is critical for $\beta$-pleated sheet formation [1]. Thus, humans but not rodents have islet amyloid deposits. Simply having the critical amino acid sequence is, however, insufficient for amyloid deposition because humans without Type II diabetes rarely develop islet amyloid [4].

To study factor(s) that could be important in islet amyloidogenesis in Type II diabetes, transgenic mice expressing the human $I A P P$ gene have been generated [1]. We observed the development of islet amyloid in our mice when fed an increased quantity of fat [5] and also found that islet amyloid developed primarily in male mice [5], an observation confirmed by others $[6,7]$. This latter finding suggests that ovarian prod- 
ucts have a protective effect on amyloid formation and is in keeping with the observation that postmenopausal women receiving oestrogen seem to be protected from developing Type II diabetes compared with their counterparts not taking oestrogen [8].

Based on these observations, we did an oophorectomy to create a state of pronounced deficiency of ovarian products in our human IAPP transgenic mice and followed them until a year of age, assessing glucose tolerance, islet beta-cell function, pancreatic peptide content and the extent of islet amyloid formation compared with sham-operated animals.

\section{Materials and methods}

Mice. Human IAPP transgenic mice were generated as described previously [5]. We studied four groups of female mice: oophorectomized and sham-operated human IAPP transgenics and oophorectomized and sham-operated non-transgenics. Mice were housed at the Seattle VA Puget Sound Health Care System (SVAPSHCS) and fed Purina Autoclavable Mouse Diet (5021; Purina Mills Inc., Richmond, Ind., USA) containing $9.0 \%$ fat $(\mathrm{w} / \mathrm{w})$. The Animal Review Committee at the SVAPSHCS approved the study.

Oophorectomy and sham surgery. Mice underwent an oophorectomy or sham surgery under pentobarbital $(100 \mathrm{mg} /$ kg i.p.) anaesthesia. For the oophorectomy, the ovarian vasculature was ligated and the ovaries excised. For the sham surgery, the ovaries were externalized and then returned to the abdominal cavity.

Intraperitoneal glucose tolerance test. After an $18 \mathrm{~h}$ fast 1 week before they were killed, mice underwent an intraperitoneal glucose tolerance test (IPGTT). Under pentobarbital $(100 \mathrm{mg} / \mathrm{kg}$ i.p.) anaesthesia, blood samples were obtained from the retroorbital sinus before and 15, 30, 60 and $120 \mathrm{~min}$ after being given dextrose $(1 \mathrm{~g} / \mathrm{kg}$ body weight of a $50 \%$ dextrose solution i.p).

Pancreas extraction. Mice were killed by i.p. injection of $200 \mathrm{mg} / \mathrm{kg}$ pentobarbital. A portion of pancreas was snap frozen in liquid nitrogen and extracted by homogenization for $10 \mathrm{~min}$ in $50 \%$ isopropanol $/ 1 \%$ trilluoracetic acid (TFA). The homogenate was stored at $-70{ }^{\circ} \mathrm{C}$ before being assayed.

Histology. Islet amyloid was detected in paraformaldehydefixed, $5 \mu \mathrm{m}$ deparaffinized pancreas sections stained with thioflavin S [5]. A minimum of six islets per section was assessed from three sections obtained from different parts of the pancreas.

Assays. Plasma was stored at $-20{ }^{\circ} \mathrm{C}$ before being assayed. Follicle stimulating hormone $(\mathrm{FSH})$ concentrations were measured by a radioimmunoassay that has intra-assay and inter-assay coefficients of variation of $9 \%$ and $13 \%$, respectively. Glucose, immunoreactive insulin (IRI), islet amyloid polypeptidelike immunoreactivity (IAPP-LI) of mouse (mIAPP-LI) and human (hIAPP-LI) were measured as described previously [9]. Protein content of the pancreatic extracts was measured using the BCA Protein Assay Kit (Pierce, Rockford, Ill., USA).
Calculations and statistical analysis. Incremental AUC above baseline for glucose and IRI responses during the IPGTT were calculated using the trapezoidal method.

Data are presented as means \pm SEM. Comparisons between groups for continuous variables were made by analysis of variance followed by a $t$ test when the data were normally distributed or the Mann-Whitney U test when they were not. Categorical data were compared using the chi squared analysis. A $p$ value of 0.05 or less was considered significant.

\section{Results}

Effect of oophorectomy on body weight, glucose tolerance and beta-cell function. Human IAPP transgenic and non-transgenic mice underwent oophorectomy or sham surgery at 6-8 weeks of age, the success of which was confirmed by measurement of FSH concentrations $6-8$ weeks after surgery. Complete follow-up studies were done on 11 oophorectomized and 8 sham-operated human IAPP transgenic mice and 7 oophorectomized and 9 sham-operated nontransgenic mice.

At 6-8 weeks of age when surgery was done, body weight was similar in all groups of mice (transgenic oophorectomy: $22.7 \pm 1.1 \mathrm{~g}$, non-transgenic oophorectomy: $21.4 \pm 0.9 \mathrm{~g}$, transgenic sham: $21.7 \pm 0.3 \mathrm{~g}$, nontransgenic sham: $21.6 \pm 1.0 \mathrm{~g}$ ). After the surgical procedure, body weight increased steadily in all groups such that at the termination of the study, body weights tended to be greater in the oophorectomized mice, but this did not reach significance (Table 1).

During IPGTTs done at 12 months of age, fasting and post-stimulation plasma glucose and IRI concentrations and the incremental responses were not different (Table 1). Thus, oophorectomy was not associated with changes in either glucose tolerance or betacell function.

Mice were killed within a week of the IPGTT. Becausae two sham-operated human IAPP transgenic mice died between the IPGTT and the planned time of killing, blood or pancreas was not obtained from them. Using the human specific IAPP assay, we found that oophorectomy was not associated with an alteration in plasma human IAPP-LI suggesting that release of this beta-cell peptide is not altered (Table 1).

Oophorectomy is associated with islet amyloid formation but not altered islet peptide content. Pancreas samples from all groups of mice were examined for islet amyloid. As expected, we observed no islet amyloid in the non-transgenic mice. Thus, for the purpose of determining whether oophorectomy was associated with an increased prevalence of islet amyloid, we included only the human IAPP transgenic mice in the analysis. The prevalence of islet amyloid was $64 \%(7 / 11)$ in the oophorectomized and $0 \%(0 / 6)$ in the sham-operated mice $(p<0.05)$. In mice with de- 
Table 1. Body weight, fasting plasma and intraperitoneal glucose tolerance test measures of glucose, IRI and human IAPP-LI at 12 months of age in female human IAPP transgenic and non-transgenic mice that had undergone oophorectomy or sham surgery

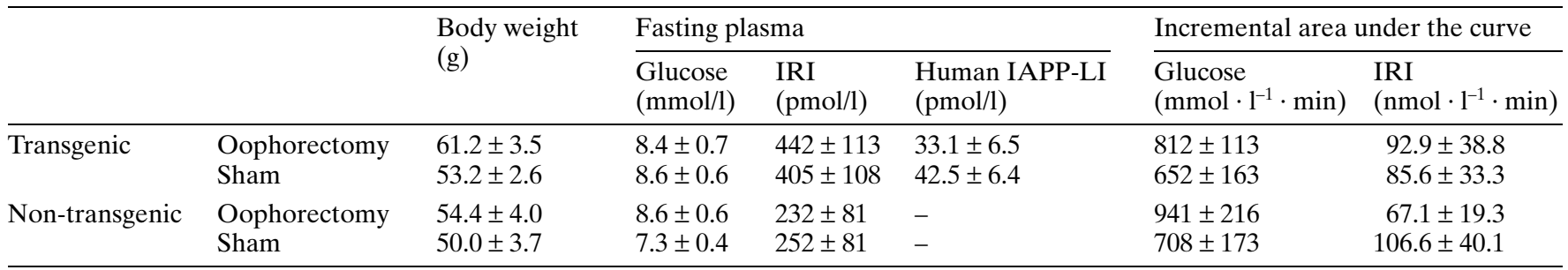

Data are means \pm SEM

Table 2. Pancreatic content of IRI, human IAPP-LI and mouse IAPP-LI at 12 months of age in female human IAPP transgenic and non-transgenic mice that had undergone oophorectomy or sham surgery

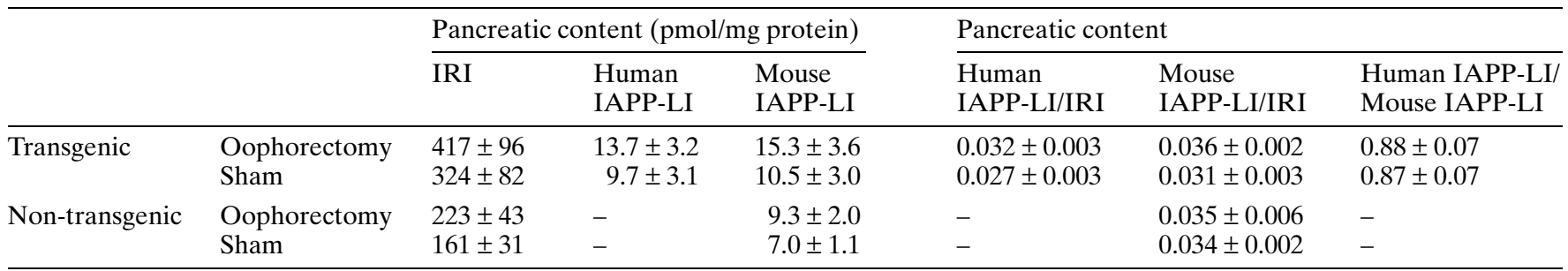

Data are means \pm SEM

tectable amyloid, most islets had at least one area of amyloid but in some islets there were extensive amyloid deposits.

Non-transgenic mice had no measurable human IAPP-LI in their pancreata and oophorectomy was not associated with a change in pancreatic content of human IAPP (Table 2). Mouse IAPP-LI was measurable in pancreata from all mice. In the transgenic mice, the concentrations of mouse and human IAPPLI were similar so that the ratio of human to mouse IAPP-LI was constant. The IRI content was not affected by oophorectomy status with the ratio between human or mouse IAPP-LI and IRI being similar. Thus, oophorectomy did not alter the pancreatic content or proportion of these three islet peptides.

\section{Discussion}

This study shows that oophorectomy in fat-fed female human IAPP transgenic mice is associated with the development of islet amyloid and that intact ovarian function protects against this deleterious effect. This finding suggests that ovarian steroids are potentially a factor in the pathogenesis of human Type II diabetes and specifically the pancreatic islet lesion of the disease. Whether the protection afforded by intact ovarian function is mediated by a direct or indirect effect of oestrogen or by a favourable balance between oestrogens and androgens cannot be discerned from study.

Although we did not identify an exact mechanism responsible for the increased prevalence of islet amy- loid in mice deficient in ovarian products, because pancreatic content and fasting plasma concentrations of human IAPP were not different in the two groups of transgenic mice, we were able to exclude the possibility that a pronounced increase in production or release of human IAPP or both was responsible. The findings of similar pancreatic content and circulating concentrations of IAPP suggests that islet amyloid formation is not dependent on increased production of IAPP, in keeping with findings that islet amyloid is not always observed in obese humans or in transgenic mice that produce and secrete increased amounts of insulin and IAPP $[1,4]$. Thus, although islet amyloid has been observed in certain lines of human IAPP transgenic mice with considerably increased IAPP production [6,7], the similar pancreatic content of human IAPP in the transgenic mice that do and do not develop amyloid highlights the importance of other factors such as dietary fat and ovarian products in islet amyloid formation.

The finding of a protective effect of ovarian products, probably oestrogen, on islet amyloidogenesis and our previous finding of a deleterious effect of dietary fat on amyloid formation is compatible with two sets of observations in humans. Firstly, increased dietary fat intake has been associated with an increased prevalence of and progression to Type II diabetes [10]. Secondly, postmenopausal women receiving oestrogen replacement therapy have a lower proportion of circulating proinsulin than their non-treated counterparts, suggesting oestrogen replenishment is associated with improved beta-cell function (unpublished observation). We therefore speculate that 
dietary fat promotes islet amyloid formation and that oestrogen is capable of reducing or modifying this effect, perhaps by a direct action on the beta-cell. Because postmenopausal oestrogen replacement is thought to be beneficial in Alzheimer's disease, a common mechanism could underlie oestrogen's protective effects in various amyloid diseases.

The role of islet amyloid in the development and progression of the hyperglycaemia of human Type II diabetes is a subject of controversy [1]. Islet amyloid is present in the majority of humans with Type II diabetes but whether it is an early event or an epiphenomenon resulting from hyperglycaemia has not been resolved. Our finding of islet amyloid in the oophorectomized mice in which glucose tolerance is not different to that of sham-operated mice and our previous observation of these deposits in male mice that are not hyperglycaemic [5], strongly suggests that the initial phases of islet amyloid formation do not require alterations in glucose tolerance. The degree of amyloidosis we observed in the oophorectomized mice did not, however, allow us to address the role of islet mass loss by amyloid in the development of hyperglycaemia.

In a mouse model of islet amyloidogenesis, we have shown that oophorectomy leads to islet amyloid formation. This finding could be of potential importance not only to understanding the pathogenesis of Type II diabetes but also has implications for the prevention and treatment of this disease and other localized syndromes associated with amyloid.

Acknowledgements. The authors are grateful to M.Abrahamson, S. Wang, C. Fernstrom, Y. McCutchen, V. Hoagland, R. Hollingsworth and D. Winch for expert technical assistance. Dr. W. Bremner is thanked for the FSH assay and Amylin Pharmaceuticals Inc. for donating reagents for measuring human IAPP. These studies were supported by National Institutes of Health (NIH) grants DK-17047 and DK-50703 and an American Diabetes Association Mentor Based Fellowship. S. Andrikopoulos was supported by a Juvenile Diabetes Foundation International Fellowship, C. B. Verchere by a Medical Research Council of Canada Postdoctoral Fellowship and J. Vidal by a Spanish Ministry of Education Post-Doctoral Fellowship.

\section{References}

1. Kahn SE, Andrikopoulos S, Verchere CB (1999) Islet amyloid: a long-recognized but underappreciated pathological feature of type 2 diabetes. Diabetes 48: 241-253

2. Westermark P, Wernstedt C, Wilander E, Hayden DW, O'Brien TD, Johnson KH (1987) Amyloid fibrils in human insulinoma and islets of Langerhans of the diabetic cat are derived from a neuropeptide-like protein also present in normal islets. Proc Natl Acad Sci USA 84: 3881-3885

3. Cooper GJ, Willis AC, Clark A, Turner RC, Sim RB, Reid KB (1987) Purification and characterization of a peptide from amyloid-rich pancreases of type 2 diabetic patients. Proc Natl Acad Sci USA 84: 8628-8632

4. Clark A, Saad MF, Nezzer T et al. (1990) Islet amyloid polypeptide in diabetic and non-diabetic Pima Indians. Diabetologia 33: 285-289

5. Verchere CB, D'Alessio DA, Palmiter RD et al. (1996) Islet amyloid formation associated with hyperglycaemia in transgenic mice with pancreatic beta cell expression of human islet amyloid polypeptide. Proc Natl Acad Sci USA 93: 3492-3496

6. Soeller WC, Janson J, Hart SE et al. (1998) Islet amyloidassociated diabetes in obese $\mathrm{A}^{\mathrm{vy}} / \mathrm{a}$ mice expressing human islet amyloid polypeptide. Diabetes 47: 743-750

7. Höppener JW, Oosterwijk C, Nieuwenhuis MG et al. (1999) Extensive islet amyloid formation is induced by development of Type II diabetes mellitus and contributes to its progression: pathogenesis of diabetes in a mouse model. Diabetologia 42: 427-434

8. Manson JE, Rimm EB, Colditz GA et al. (1992) A prospective study of postmenopausal oestrogen therapy and subsequent incidence of non-insulin-dependent diabetes mellitus. Ann Epidemiol 2: 665-673

9. Andrikopoulos S, Verchere CB, Teague JC et al. (1999) Two novel immortal pancreatic $\beta$-cell lines expressing and secreting human islet amyloid polypeptide do not spontaneously develop islet amyloid. Diabetes 48: 1962-1970

10. Tsunehara CH, Leonetti DL, Fujimoto WY (1990) Diet of second-generation Japanese-American men with and without non-insulin-dependent diabetes. Am J Clin Nutr 52: 731-738 